\title{
Envolvimento paterno: validade interna da versão brasileira do Inventory of Father Involvement
}

\author{
Ligia de Santis ${ }^{1}$, Elizabeth Joan Barham \\ Universidade Federal de São Carlos, São Carlos-SP, Brasil \\ Susana Coimbra, Anne Marie Germaine Victorine Fontaine \\ Universidade do Porto, Porto, Portugal
}

\section{RESUMO}

Existem evidências extensas que apontam a importância da relação pai-filho para o desenvolvimento e o bem-estar dos filhos, das mães e dos próprios pais. O envolvimento paterno é um construto multidimensional que inclui envolvimentos diretos e indiretos do pai com o filho. Para avançar nessa área, no Brasil, é preciso que tenhamos medidas válidas desse construto, tal como o Inventário de Envolvimento Paterno (Inventory of Father Involvement - IFI). O objetivo do presente estudo foi investigar a estrutura fatorial e confiabilidade interna da versão brasileira do IFI (IFI-BR). Um questionário sociodemográfico e o IFI-BR foram aplicados em 200 homens com filhos entre cinco e dez anos de idade. Os resultados indicam evidências de validade interna para o IFI-BR, confirmando uma estrutura fatorial muito similar à do IFI, composta por oito dos nove fatores originais de primeira ordem e um fator geral de envolvimento paterno, de segunda ordem, com uma confiabilidade interna adequada para esses oito fatores.

Palavras-chave: envolvimento paterno; medida; validação.

ABSTRACT - Father involvement: Internal validity of the Brazilian version of the Inventory of Father Involvement Extensive evidence points to the importance of father-child relationship in the development and for the wellbeing of children, mothers and fathers. Father involvement is multidimensional and includes direct and indirect father-child involvements. In order to progress in this area, a valid measure of this construct, like the Inventory of Father Involvement (IFI), is needed in Brazil. The objective of this study was to investigate the structure of factors and internal reliability of the Brazilian version of IFI (IFI-BR). A sociodemographic questionnaire and the IFI-BR were filled in by 200 men who had five- to ten-year-old children. The results prove internal validity of the IFI-BR and confirms a structure of factors very similar to that of the original IFI, including eight of the nine original first-order factors and a general second-order factor, with indicators of internal reliability that were adequate for these eight factors.

Keywords: father involvement; measure; validation.

RESUMEN - Envolvimiento paterno: Validez interna de la versión brasileña del Inventory of Father Involvement Existen amplias evidencias que apuntan a la importancia del envolvimiento paterno en el desarrollo y el bienestar de los hijos, las madres y los padres. El envolvimiento paterno es un constructo multidimensional, incluyendo envolvimientos directos e indirectos del padre con el hijo. Para avanzar en esta área, en Brasil, es necesario tener una medida válida de este constructo, como el Inventory of Father Involvement (IFI). El objetivo de este estudio fue investigar la estructura factorial y la fiabilidad interna de la versión brasileña del IFI (IFI-BR). Un cuestionario sociodemográfico y el IFI-BR se aplicaron en 200 hombres con hijos entre cinco y diez años de edad. Los resultados evidencian la validez interna del IFI-BR, confirmando una estructura factorial muy similar a la del IFI, compuesta por ocho de los nueve factores de primer orden originales y un factor de segunda orden general, con adecuada fiabilidad interna de estos ocho factores.

Palabras clave: envolvimiento paterno; medida; validación.

No Brasil, assim como em outros países, o papel paterno vem se modificando ao longo do tempo, impulsionado por mudanças nas estruturas e dinâmicas familiares (Bueno \& Vieira, 2014; Gomes, Bossardi, Cruz, Crepaldi, \& Vieira, 2014; Seward \& Stanley-Stevens, 2014). Inicialmente, em função da divisão de tarefas baseadas em gênero, esperava-se do pai o sustento financeiro da família (pai tradicional). Com o tempo, esse ideal modificou-se, sendo incluídas ao modelo paterno características de um pai mais próximo psicologicamente e mais presente (pai moderno) (Silva, 2011), mas que ainda não participava significativamente nos cuidados 
diretos dos filhos. Hoje em dia, novas categorias de envolvimento estão sendo acrescentadas, construindo um ideal no qual o pai participa de atividades relacionadas ao cuidado da casa e dos filhos, dividindo, de forma mais equitativa, essas funções com a mãe da criança (pai emergente ou polivalente) (Santis, 2012; Silva, 2011).

Assim como o ideal acerca do papel paterno, a definição do envolvimento paterno também evoluiu ao longo do tempo. Desde que o termo surgiu na literatura, quando era mensurado pela comparação da presença versus ausência do pai no ambiente doméstico (Cia, 2009), diversas conceituações da relação pai-filho foram elaboradas. Daniel Paquette, por exemplo, observou diferenças entre pais e mães nos seus padrões de interação com os filhos a fim de caracterizar as contribuições específicas que tornam a presença do pai importante. Com base em observações como o fato de os homens se envolverem mais do que as mulheres em brincadeiras físicas com os filhos, Paquette teorizou o apego entre pai e filho, a partir do conceito de relação de ativação, que enfatiza o papel do pai na abertura social do filho para se relacionar com outras pessoas (Paquette \& Dumont, 2013; Stevenson \& Crnic, 2013). Assim como este autor, Michael Lamb (1997) parte do princípio de que, além do tempo de interação entre pai e filho, a forma como interagem também é relevante. Lamb propôs uma conceituação do envolvimento paterno ainda mais abrangente, explicitando e unindo conceitos discutidos na literatura em três dimensões:

1. interação (contatos diretos entre pai e filho);

2. acessibilidade (disponibilidade, física e psicológica, do pai para a criança); e

3. responsabilidade (pai responsável pelos cuidados que o filho recebe e por promover seu desenvolvimento e bem-estar).

Nesse mesmo sentido, Pleck $(2010 ; 2012)$ ampliou ainda mais essa conceituação, propondo critérios para descrever o relacionamento paterno de boa qualidade:

- engajamento positivo em atividades (participar de atividades de forma a favorecer o desenvolvimento do filho);

- carinho e responsividade (demonstrar afeto e responder às necessidades do filho);

- controle (lidar bem com situações difíceis nas interações com o filho);

- cuidado indireto, tanto social (promover o repertório social da criança) quanto material (promover bens e serviços importantes para o desenvolvimento infantil); e

- responsabilidade (perceber e tomar medidas para suprir as necessidades da criança).

Por isso, o conceito de envolvimento paterno é, atualmente, considerado multidimensional (Arrais, 2012; Lima \& Rodrigues, 2012; Tautolo, Schluter, \&
Paterson, 2015). Assim, além da dimensão do sustento financeiro, entende-se que o relacionamento pai-filho inclui componentes afetivos, cognitivos e éticos, bem como componentes comportamentais observáveis diretos com o filho (interações), ou indiretos (por exemplo, suporte psicológico à mãe) (Hawkins et al., 2002; Pleck, 2007).

Diante do aumento do interesse no estudo do envolvimento paterno (Bueno \& Vieira, 2014; Volker, 2014) e, consequentemente, no número de trabalhos sobre o tema (Barrocas, Vieira-Santos, Paixão, Roberto, \& Pereira, 2016), nota-se que a atuação do pai em relação ao filho pode acarretar consequências de grande importância para o bem-estar e o desenvolvimento, tanto do filho (Allen \& Daly, 2007; Cabrera, Shannon, \& TamisLeMonda, 2007; Gaumon, Paquette, Cyr, ÉdmondNajamura, \& St-André; Pleck, 2012; Santis \& Barham, no prelo; Sarkadi, Kristiansson, Oberklaid, \& Bremberg, 2008; Stevenson \& Crnic, 2013) quanto do próprio pai (Allen \& Daly, 2007; Bartlett, 2004; Pleck, 2012; Santis \& Barham, no prelo) e para a qualidade da relação conjugal que este estabelece (Mehall, Spinrad, Eisenberg, \& Gaertner, 2009; Santis \& Barham, no prelo; Simões, Leal, \& Marôco, 2010).

\section{Avaliação do envolvimento paterno}

Apesar de já evidenciada a relevância do envolvimento paterno, ainda se nota a falta de intervenções direcionadas para o aprimoramento específico da atuação do pai junto a seu filho (Benzies, Magil-Evans, Hayden, \& Ballantyne, 2013; Flippin \& Crais, 2011), bem como a fragilidade metodológica desses programas, quando existentes (Bronte-Tinkew, Burkhauser, \& Metz, 2012). Embora recentemente algumas intervenções para pais (homens) já tenham sido criadas, estas, no entanto, não contam com robustos procedimentos de avaliação do envolvimento paterno (Bronte-Tinkew et al., 2007; Lundahl, Tollefson, Risser, \& Lovejoy, 2008; McAllister, Burgess, Kato, \& Barker, 2012). Paschoalick (2008), por meio de uma revisão da literatura internacional, examinou diferentes instrumentos de envolvimento paterno e destacou o Inventário de Envolvimento Paterno (Inventory of Father Involvement - IFI) de Hawkins et al. (2002), uma vez que ele apresenta:

- fundamentação teórica pautada em estudos recentes sobre o conceito de envolvimento paterno;

- informações sobre sua estrutura interna, coerentes com essa visão atual do construto; e, além disso,

- por ser um instrumento de autorrelato de rápida aplicação.

Em estudo de validação nos Estados Unidos, o IFI foi respondido por 723 pais de crianças entre cinco e dez anos. Por meio de uma análise fatorial exploratória, Hawkins et al. (2002) identificaram no instrumento nove fatores distintos, sendo eles: 
1. disciplina e ensino de responsabilidade: por exemplo, estabelecer regras e limites para o comportamento do filho;

2. encorajamento escolar: encorajar seu filho a ter sucesso na escola;

3. suporte à mãe: cooperar com a mãe de seu filho na criação deste;

4. sustento: suprir as necessidades básicas do filho;

5. tempo juntos e conversas: passar tempo com o filho fazendo coisas que ele goste;

6. elogios e afeto: elogiar seu filho por ser bem-comportado ou fazer a coisa certa;

7. desenvolvimento de talentos e interesses futuros: planejar-se para o futuro do filho;

8. leitura e ajuda com tarefas escolares: ler para o filho; e

9. acompanhamento: comparecer aos eventos nos quais o filho participa.

A fim de se obter uma análise mais rigorosa da estrutura interna do IFI, por meio da análise fatorial confirmatória (AFC), Hawkins et al. (2002) testaram dois modelos fatoriais principais, ambos com estruturas de segunda ordem. Um desses modelos apresentava um único fator geral de segunda ordem, englobando os demais fatores; o outro, por sua vez, apresentava dois fatores de segunda ordem, agrupando os que capturavam aspectos mais "tradicionais" de envolvimento (como "sustento") e aqueles que traziam ideias mais contemporâneas, dos "novos pais" (como "leitura e ajuda com tarefas escolares"). Segundo os autores, apesar das poucas diferenças encontradas entre os índices de ajuste dos modelos testados, o exemplar com um único fator geral de segunda ordem apresentou os melhores índices, além de representar, de forma mais parcimoniosa, o envolvimento paterno. A análise da consistência interna indicou valores adequados para as nove subescalas do IFI, e a estrutura fatorial mostrou-se, portanto, coerente com a concepção teórica multidimensional de envolvimento paterno, apresentada pelos autores (Hawkins et al., 2002).

Em dois estudos de validação do IFI para Portugal, Barrocas et al. (2016) encontraram altos índices de confiabilidade interna para o escore global da versão portuguesa do instrumento $(0,93$ e 0,95$)$. Os autores também confirmaram uma estrutura com nove fatores de primeira ordem, por meio de uma AFC, além de obterem evidências iniciais de validade concorrente e discriminante.

Por meio de uma revisão da literatura acerca dos instrumentos de avaliação do envolvimento paterno no Brasil, Gomes et al. (2014) evidenciaram que o uso de instrumentos de coleta de dados não validados ainda é uma prática relativamente comum, diante da carência de medidas com propriedades psicométricas adequadas, o que indica a importância de investir na validação de um instrumento dessa natureza. Paschoalick (2008; 2009) iniciou o processo de adaptação e validação do IFI para uso no Brasil, cumprindo as etapas de tradução, retrotradução e avaliação por parte de juízes. Em um estudo com 199 pais de crianças em idade pré-escolar, Paschoalick (2009) relatou que a consistência interna do IFI-BR, para todos os itens da escala, era alta $(\alpha=0,89)$. A autora realizou somente uma análise fatorial exploratória — não uma $\mathrm{AFC}$ - para verificar se as dimensões do IFI correspondiam às do IFI-BR. A dimensão "desenvolvimento de talentos e interesses futuros" não apareceu entre os pais que participaram deste estudo.

No presente trabalho, portanto, o objetivo foi testar a coerência entre o construto multidimensional de envolvimento paterno de Hawkins et al. (2002) e a estrutura interna do IFI-BR, quando respondido por pais brasileiros, e avaliar a consistência das escalas do instrumento. Mais especificamente, foram investigadas:

- a confiabilidade interna dos fatores do IFI-BR; e

- a estrutura fatorial do instrumento.

\section{Método}

\section{Participantes}

Foram incluídos no estudo pais (homens) de crianças de cinco a dez anos de idade, que mantinham contato com seu filho pelo menos uma vez por semana. Dos 208 participantes, foram excluídos dados de pais que:

- demonstraram não entender como usar as escalas de pontuação (cinco pais); ou

- estavam acompanhados pela mãe da criança, que influenciava em suas respostas (de dois pais).

Atentou-se, também, para a possibilidade de haver observações extremas (outliers), muito afastadas das restantes. Quando considerado o escore global de envolvimento paterno, foi retirado um participante que apresentou um valor extremo. A amostra final foi de 200 pais. Destes, 80,5\% foram recrutados em São Carlos (cidade com cerca de 220.000 habitantes), e 19,5\%, em Itápolis (cidade com aproximadamente 40.000 habitantes). Por não ser uma amostra aleatória, para evitar um problema de homogeneidade da composição da amostra, procuraram-se pais de estudantes de escolas públicas e particulares. Do total, 43,5\% dos filhos estudavam em escolas públicas e 56,5\%, em escolas privadas. Maiores informações sobre os participantes são apresentadas na Tabela 1.

\section{Instrumentos}

Inventário de envolvimento paterno. A versão original do instrumento (Hawkins et al., 2002) foi traduzida e algumas evidências de validade já foram analisadas (Paschoalick, 2009). O instrumento inclui 26 itens e é utilizado para avaliar nove dimensões do envolvimento paterno. O pai autoavalia a qualidade do envolvimento com seu filho-alvo para cada item, usando uma escala de pontuação, tipo Likert, de 7 pontos, que 
varia de 0 (muito pobre) a 6 (excelente). Os pais que não praticam a atividade - em função da idade do filho, por exemplo - podem marcar "não se aplica".

Questionário sociodemográfico. Foram solicitadas informações sobre o perfil sociodemográfico do respondente e sua família, como estado conjugal e renda familiar.

\section{Procedimento de coleta de dados}

Inicialmente, foi feito contato com o responsável pela Secretaria Municipal de Educação nas cidades de São Carlos e Itápolis e com a diretoria de diversas escolas particulares desses lugares, para obter autorização para contatar pais de alunos. Os diretores de sete escolas públicas e de sete particulares de São Carlos e de uma escola pública e uma particular de Itápolis aceitaram contribuir com o estudo.

Após a autorização da direção das escolas, todos os pais de alunos entre cinco e dez anos de idade receberam, por meio de seus filhos, uma carta-convite para participar do estudo. A taxa de participação variava, mas em todas as escolas foi abaixo de $21 \%$ (a taxa média foi de cerca de $8 \%$ ). No momento combinado com os pais, a pesquisadora apresentava-se, descrevia os objetivos gerais da pesquisa, pedia para que os pais lessem e assinassem o Termo de Consentimento Livre e Esclarecido e entregava os instrumentos. A pesquisadora assistia os pais com dificuldades de compreensão dos itens. Esse encontro durava, em média, 45 minutos. Em seguida, era entregue para cada pai um folheto com informações da literatura científica sobre a importância do relacionamento entre pais e filhos. Ao final do estudo, um resumo dos resultados principais, em linguagem acessível, foi enviado aos participantes que o solicitaram e que informaram seu e-mail. Também foi disponibilizada uma cópia impressa desse material para as escolas envolvidas.

\section{Cuidados éticos}

Foram tomados todos os cuidados para a realização de pesquisas com seres humanos, em acordo com a Resolução $n^{\circ} 466$, de 12 de dezembro de 2012. O projeto foi aprovado pelo Comitê de Ética para Pesquisas com Seres Humanos da Universidade Federal de São Carlos (número do parecer: 699.911).

\section{Procedimento de análise dos dados}

Os dados foram analisados usando o programa SPSS-20 para Windows. As distribuições de valores para os itens, os fatores e o escore global do IFI-BR foram examinadas para verificar se essas atendiam aos critérios de uma distribuição normal. Também foi verificado se mais de $80 \%$ dos pais respondiam a algum item de forma idêntica (Clark \& Watson, 1995). Tanto a normalidade da distribuição quanto a variabilidade das respostas - ou sensibilidade do teste - estavam adequadas (Kline, 2011; Marôco, 2010).

Validade interna. Tendo em vista que existe um modelo teórico multifatorial de envolvimento paterno, confirmado pela estrutura fatorial do IFI na sociedade americana, realizou-se uma AFC (Marôco, 2010) para testar o modelo fatorial de Hawkins et al. (2002). Antes de proceder à AFC, foram calculados os índices de consistência interna (alfa de Cronbach) para cada um dos nove fatores (ou subescalas), conforme a estrutura fatorial apresentada no estudo americano. No mínimo, espera-se que o número de respondentes para realizar uma AFC seja de cinco a dez pessoas por item (Tornimbeni, Pérez, \& Olaz, 2008). Este estudo atende esse critério. A qualidade dos modelos foi inicialmente avaliada verificando se os pesos fatoriais padronizados dos itens nos fatores eram superiores a 0,40. A fim de avaliar a qualidade do seu ajustamento global, foram utilizados os índices sugeridos por Kline (2011) e Schweizer (2010), assim como os seguintes valores de ajustamento considerados aceitáveis: razão $\chi^{2} /$ g.1. $<5$; Bentler Comparative Fit Index (CFI) >0,90; Root Mean Square Error of Approximation (RMSEA) $<0,08$; e Standardized Root Mean Square Residual (SRMR) $<0,08$ (Hooper, Coughlan, \& Mullen, 2008; Hu \& Bentler, 1999; Kline, 2011; Marsh, Hau, \& Wen, 2009; Schweizer, 2010; Tabachnick \& Fidell, 2007).

\section{Resultados}

\section{Consistência interna}

Embora seja comum definir valores ideais para alfa de Cronbach como aqueles entre 0,70 e 0,90, alguns

Tabela 1

Características dos participantes

\begin{tabular}{lcccc}
\hline & Mínimo & Máximo & Média & DP \\
\hline Número de filhos & 1 & 6 & 1,94 & 0,84 \\
Idade do filho-alvo $^{1}$ & 5 & 10 & 7,255 & 1,8 \\
Idade do pai $^{\text {Renda familiar (R\$) }}$ & 22 & 70 & 39,5 & 7,42 \\
Tempo de união (em anos) $^{2}$ & 200,00 & 70000,00 & 7535,18 & 8521,08 \\
\hline
\end{tabular}

Nota: DP=desvio-padrão; ${ }^{1}$ Caso tivesse mais de um filho, o pai deveria responder aos instrumentos considerando seu filho mais velho de até dez anos (filho-alvo); ${ }^{2}$ Considerando apenas os pais que descreveram estar casados ou vivendo como casados. 
autores defendem que, na área das Ciências Sociais, valores acima de 0,60 podem ser considerados aceitáveis (Kline, 2011; Marôco \& Garcia-Marques, 2006). Com exceção da subescala "Desenvolvimento de talentos e interesses futuros" $(\alpha=0,51)$, a consistência interna das demais subescalas foi adequada, variando de 0,65 a 0,81 . Observou-se que, diferentemente dos demais itens, os dessa subescala apresentaram altas taxas de dados omissos $(12,46 \%)$, com taxas maiores entre os pais de crianças mais novas, em comparação com os que tinham filhos mais velhos. Aparentemente, muitos dos pais não avaliaram seu desempenho nessas atividades por considerá-las irrelevantes. Optou-se, portanto, por excluir esses três itens - e, consequentemente, essa subescala - da AFC.

\section{Análise fatorial confirmatória}

Nessa etapa, o instrumento analisado incluía 23 itens e oito fatores. Os modelos testados para a estrutura fatorial da amostra de pais brasileiros foram os mesmos dois descritos na análise fatorial apresentada por Hawkins et al. (2002), diferenciando destes, apenas, na exclusão do fator "Desenvolvimento de talentos e interesses futuros". No Modelo 1, que é o que Hawkins et al. adotaram ao final de sua análise, existe um único fator geral de segunda ordem, englobando todos os fatores, indicando que pais mais envolvidos participam igualmente em todas as atividades descritas. No Modelo 2, existem dois fatores de segunda ordem: um deles representa atividades de apoio indireto, como a preocupação com o sustento e a educação do filho. Já o outro capta atividades típicas de um envolvimento direto, em que o pai se envolve afetivamente e na rotina de cuidados do filho. Pais tradicionais apresentariam um envolvimento maior apenas com atividades indiretas, enquanto pais com um relacionamento moderno ou polivalente deveriam realizar atividades dos dois conjuntos (indireto e direto). Na Tabela 2, são apresentados os indicadores do ajuste desses dois modelos aos dados coletados com os pais brasileiros.

Foram comparados os valores obtidos para cada indicador de ajuste com os de referência, para cada modelo. Os pesos fatoriais e três dos índices de ajustamento global foram adequados. Como o valor do CFI estava próximo ao de referência, a introdução de pequenos ajustes nos modelos para atingir o critério se justifica, permitindo prosseguir com a análise desses dois modelos. No entanto, foi encontrado um problema no Modelo 2. Havia uma correlação alta $(r=0,82)$ entre os dois fatores de segunda ordem, indicando a ampla sobreposição entre os fatores que representam o envolvimento paterno indireto ("tradicional") e o direto ("novos pais"). Portanto, é desnecessário incluir dois fatores de segunda ordem, o que justifica a introdução de ajustes na estrutura interna do IFI-BR, tendo como base a estrutura do Modelo 1.

\section{Estrutura fatorial confirmatória final}

Uma vez que um dos índices de qualidade de ajuste global (CFI) do modelo fatorial adotado para a confirmação da estrutura interna do IFI-BR ainda não apresentava um valor superior a 0,90 , optou-se por permitir a introdução de três correlações entre erros, de modo a evitar a exclusão de itens adicionais, que seria prejudicial para a validação futura do instrumento com outras amostras e para, na medida do possível, conservar intacto o conteúdo teórico do mesmo. A escolha dessas ligações baseou-se no valor dos índices de modificação e nas semelhanças observadas no conteúdo desses itens. Ligações entre erros sugerem haver uma associação entre dois itens em relação à parte da variância de cada um deles que não explica o fator ao qual estão ligados (Hooper et al., 2008). Assim, foram correlacionados os erros dos itens 5 ("Encorajar seu filho a fazer suas tarefas escolares") e 23 ("Ajudar seu filho com tarefas escolares"), 17 ("Dizer ao seu filho que você o ama") e 12 ("Ser amigo de seu filho") e 23, de novo, e 25 ("Estar envolvido na rotina diária e regular de tomar conta de seu filho e de suprir suas necessidades básicas").

$\mathrm{Na}$ última linha da Tabela 2, são apresentados os valores de ajustamento para o modelo final, assim transformado, incluindo as três ligações entre erros. Na Figura 1, é apresentada a estrutura desse modelo final. A consistência interna de dois fatores ("Leitura e ajuda com tarefas escolares" e "Acompanhamento") foi satisfatória (0,674 e 0,652, respectivamente), e dos demais fatores, boa $(\alpha \geq 0,70)$.

Tabela 2

Índices de ajustamento para os Modelos 1, 2 e final

\begin{tabular}{|c|c|c|c|c|c|}
\hline & \multirow{2}{*}{ Pesos fatoriais } & \multicolumn{4}{|c|}{ Índice } \\
\hline & & $\chi^{2} / g .1$. & CFI & RMSEA & SRMR \\
\hline Valor de referência & $>0,40$ & $<5$ & $>0,90$ & $<0,08$ & $<0,08$ \\
\hline Modelo 1 & $\geq 0,51$ & 2,108 & 0,871 & 0,075 & 0,070 \\
\hline Modelo 2 & $\geq 0,51$ & 2,034 & 0,880 & 0,072 & 0,069 \\
\hline Modelo final & $\geq 0,59$ & 1,857 & 0,901 & 0,066 & 0,067 \\
\hline
\end{tabular}

Nota: CFI=Bentler Comparative Fit Index; RMSEA=Root Mean Square Error of Approximation; SRMR=Standardized Root Mean Square Residual. 


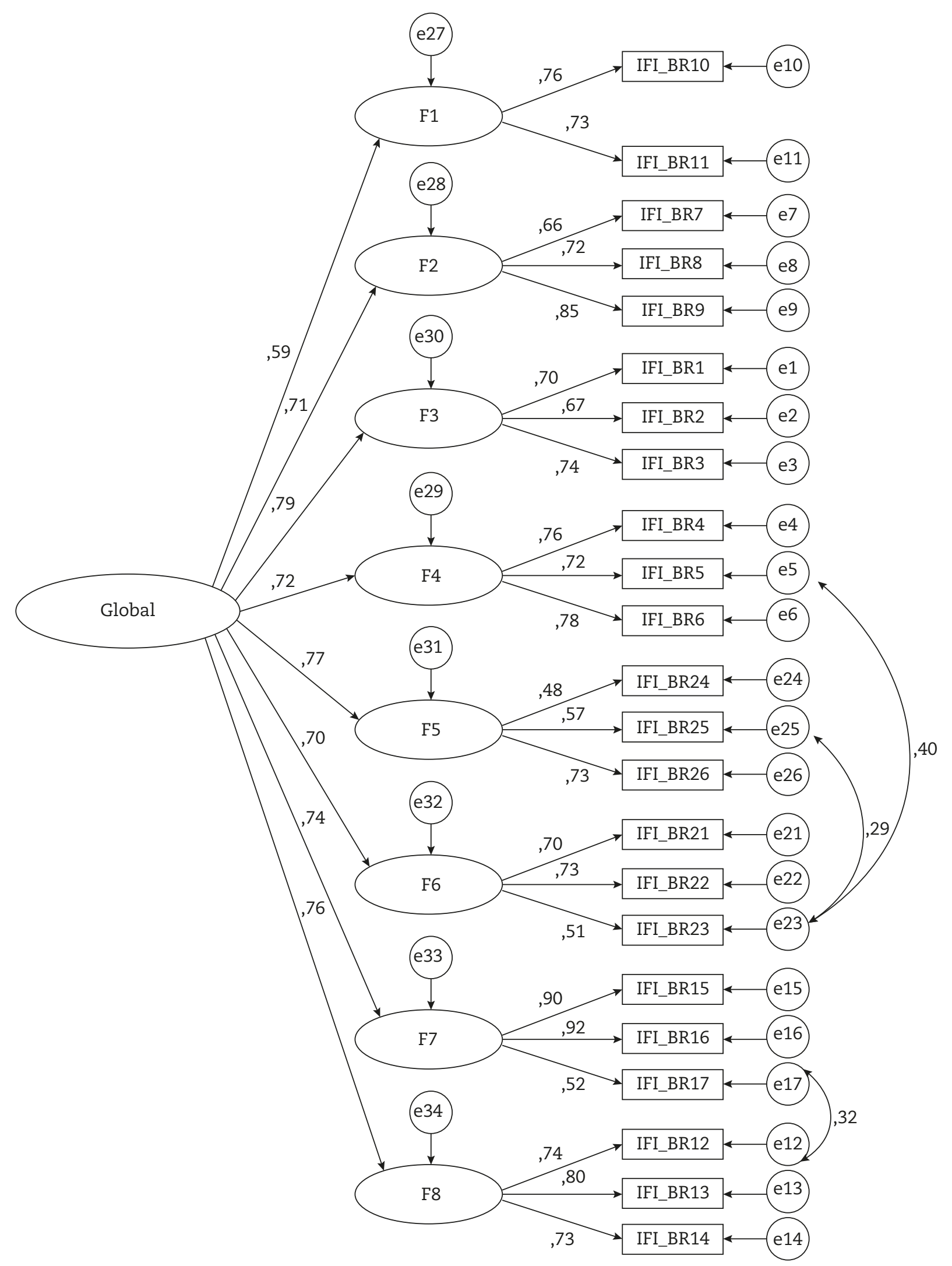

Nota: F1=Fator Sustento; F2= Fator Suporte à mãe; F3= Fator Disciplina e ensino de responsabilidade; F4= Fator Encorajamento escolar; F5= Fator Acompanhamento; F6= Fator Leitura e ajuda com tarefas escolares; F7= Fator Elogios e afeto; F8= Fator Tempo juntos e conversas.

Figura 1. Estrutura fatorial final do modelo adotado para este estudo 


\section{Discussão}

Na presente pesquisa, foram encontradas evidências de validade interna para o IFI-BR, quando aplicado em uma amostra de pais (homens) brasileiros, com filhos entre cinco e dez anos. Os resultados obtidos com a amostra brasileira apoiam a proposição de que o envolvimento paterno seja um construto multidimensional, composto por oito fatores com confiabilidade interna adequada, representando as diversas esferas de atuação paterna, todos ligados a um único fator de segunda ordem. Essa visão sobre o envolvimento paterno é sustentada por teóricos recentes da área, tais como Lamb (1997) e Paquette e Dumont (2013), que superaram a ideia de que esse tipo de relacionamento seria pautado unicamente na presença do pai ou no tempo de interação observável deste com o filho, e, ainda, passaram a entendê-lo como composto por diversas esferas distintas (Hawkins et al., 2002; Pleck, 2010; 2012).

Além disso, os resultados do presente estudo, referente à validação brasileira do IFI, somam-se aos achados de Barrocas et al., (2016), sobre a validação do IFI para a população portuguesa, indicando a validade dessa medida em diferentes contextos culturais. Foram notadas semelhanças entre as estruturas fatoriais confirmadas nesses estudos, ambas apontando para um envolvimento paterno multifatorial, sendo que oito dos nove fatores da estrutura americana e portuguesa foram confirmados na amostra brasileira. Além de respaldo teórico, a confirmação, com pais brasileiros, de uma estrutura fatorial muito similar à estrutura obtida com pais estadunidenses e portugueses permitirá que esse instrumento seja utilizado para comparar o envolvimento de pais de diferentes culturas.

Destaca-se que a confiabilidade interna encontrada foi adequada para oito dos nove fatores originalmente estabelecidos por Hawkins et al. (2002). O fator "Desenvolvimento de talentos e interesses futuros", por apresentar confiabilidade interna inadequada no presente estudo, foi excluído da AFC. Comparando esses resultados brasileiros com a análise fatorial exploratória apresentada por Paschoalick (2009) - com uma amostra de 199 pais brasileiros com filhos de três a cinco anos de idade -, nota-se que os itens que compõem esse fator também não se comportaram da maneira observada na amostra americana de Hawkins et al. (2002).

Uma hipótese que poderia explicar essa diferença é que, para os pais brasileiros, o envolvimento em atividades que promovem o desenvolvimento de talentos e interesses dos filhos - como representadas no IFI-BR - surge mais tardiamente, em comparação com os pais estadunideneses. Outra é que os itens desse fator do IFI não descrevem a forma como os pais brasileiros se envolvem nessa área. Apesar da exclusão desse fator, na análise confirmatória realizada para este estudo, a relevância do envolvimento do pai nessa esfera da vida de seu filho é inquestionável. Assim, o investimento em pesquisas adicionais dessa escala é aconselhado, recomendando-se, no entanto, a manutenção dos três itens que compõem o fator excluído, por possuírem importância teórica, uma vez que o IFI-BR foi respondido por poucas pessoas, já que ainda está em processo de validação no contexto brasileiro. Paralelamente e para melhor compreender a diferença observada, seria interessante, em pesquisas futuras, explorar as crenças de pais brasileiros sobre como e quando é importante investir no desenvolvimento dos talentos de seus filhos.

Pensando nas próximas etapas do processo de validação do IFI-BR para o contexto brasileiro, ainda será importante:

- avaliar a estabilidade temporal desse instrumento;

- verificar evidências de validade externa, correlacionando escores no IFI-BR e indicadores de desenvolvimento e bem-estar de pais, mães e filhos;

- verificar a relação entre escores no IFI-BR e dados observacionais sobre a qualidade do envolvimento paterno;

- realizar uma AFC com uma segunda amostra de pais brasileiros; e

- normatizar os resultados, usando uma terceira amostra, que precisaria ser grande e representativa da população brasileira.

Finalmente, considerando a importância de acompanhar o envolvimento de pais de crianças menores de cinco anos de idade, sugere-se, ainda, verificar a possibilidade de usar esse instrumento junto a pais de crianças mais novas. Para isso, é necessário atentar-se a especificidades da fase de desenvolvimento do filho, que podem afetar o envolvimento de pais em diferentes áreas da vida da criança. A validação de instrumentos de medida no Brasil, para avaliar o envolvimento de pais (homens) com seus filhos de diferentes idades, será muito importante para o desenvolvimento de pesquisas consistentes sobre o tema, representando um avanço científico importante. Além disso, um instrumento validado para medir o envolvimento paterno será útil para a prática clínica baseada em evidências, por contribuir para a avaliação de intervenções que promovam interações entre pais e filhos que resultam em um envolvimento paterno de boa qualidade.

\section{Agradecimentos}

Reconhecemos e agradecemos o apoio financeiro das agências de fomento: Coordenação de Aperfeiçoamento de Pessoal de Nível Superior (CAPES) e Fundação de Amparo à Pesquisa do Estado de São Paulo (FAPESP), processo n ${ }^{\circ}$ 2014/01671-9. 


\section{Referências}

Allen, S., \& Daly, K. (2007). The effects of father involvement: an updated research summary of the evidence. Father Involvement Research Alliance. University of Guelph, Ontario, Canada.

Arrais, A. I. L. (2012). Envolvimento paterno, stress parental e o apoio social em pais de crianças em idade escolar. Dissertação de mestrado apresentada ao programa de Mestrado Integrado em Psicologia da Universidade de Lisboa, Portugal.

Barrocas, J., Vieira-Santos, S., Paixão, R., Roberto, M. S., \& Pereira, C. R. (2016). The "Inventory of Father Involvement-Short Form" Among Portuguese Fathers: Psychometric Properties and Contribution to Father Involvement Measurement. Psychology of Men E Masculinity, 18, 144-156. doi: 10.1037/men0000050

Bartlett, E. E. (2004). The effects of fatherhood on the health of men: a review of the literature. Journal of Men's Health and Gender, 1(2), 159-169. doi: 10.1016/j.jmhg.2004.06.004

Benzies, K. M., Magil-Evans, J. E., Hayden, K. A., \& Ballantyne, M. (2013). Key components of early intervention programs for preterm infants and their parents: A systematic review and meta-analysis. BMC Pregnancy and Childbirth. 13(1), S10. doi: 10.1186/1471-239313-S1-S10

Bronte-Tinkew, J., Burkhauser, M., \& Metz, A. J. R. (2012). Elements of promising practices in fatherhood programs: evidence-based research findings on interventions for fathers. Fathering, 10(1), 6-30. doi: 10.3149/fth.1001.6

Bronte-Tinkew, J., Carrano, J. B. S., Allen, T. M. S. W., Bowie, L. M. A., Mbawa, K. M. A., \& Matthews, G. B. A. (2007). Elements of promising practice for fatherhood programs: Evidence-based research findings on programs for fathers. U.S. Department of Health and Human Services, Office of Family Assistance. Washington, D.C., USA.

Bueno, R. K., \& Vieira, M. L. (2014). Análise de estudos brasileiros sobre o pai e o desenvolvimento infantil. Psicologia Argumento, 32(76), 151-159. doi: 10.7213/psicol.argum.32.076.AO10

Cabrera, N. J., Shannon, J. D., \& Tamis-LeMonda, C. (2007). Fathers' influence on their children's cognitive and emotional development: from toddlers to Pre-K. Applied Development Science, 11(4), 208-213. doi: 10.1080/10888690701762100

Cia, F. (2009). Um programa para aprimorar o envolvimento paterno: impactos no desenvolvimento do filho. Tese de doutorado apresentado ao Programa de Pós-Graduação em Educação Especial da Universidade Federal de São Carlos, São Carlos, SP, Brasil.

Clark, L. A., \& Watson, D. (1995). Constructing validity: basic issues in objective scale development. Psychological Assessment, 7(3), $309-319$. Recuperado de http://www3.nd.edu/ ghaeffel/Clark\&Watson(1995).pdf

Flippin, M., \& Crais, E. R. (2011). The need for more effective father involvement in early autism intervention: a systematic review and recommendations. Journal of Early Intervention, 33(1), 24-50. doi: 10.1177/1053815111400415

Gaumon, S., Paquette, D., Cyr, C., Édmond-Najamura, M., \& St-André, M. (2016). Anxiety and attachment to the mother in preschoolers receiving psychiatric care: The father-child activation relationship as a protective care. Infant Mental Health Journal, 37(4), 372-387. doi: 10.1002/imhj.21571

Gomes, L. B., Bossardi, C. N., Cruz, R. M., Crepaldi, M. A., \& Vieira, M. L. (2014). Propriedades psicométricas de instrumentos de avaliação do envolvimento paterno: revisão de literatura. Avaliação Psicológica, 13(1), 19-27.

Hawkins, A. J., Bradford, K. P., Palkovitz, R., Christiansen, S. L., Day, R. D., \& Call, V. R. A. (2002). The inventory of father involvement: a pilot study of a new measure of father involvement. The Journal of Men's Studies, 10(2), 183-196. doi: 10.3149/jms.1002.183

Hooper, D., Coughlan, J., \& Mullen, M. R. (2008). Structural equation modelling: Guidelines for determining model fit. The Electronic Journal of Business Research Methods, 6, 53-60. Recuperado de http://arrow.dit.ie/cgi/viewcontent.cgi?article=1001\&context=buschmanart

Hu, L., \& Bentler, P. M. (1999). Cutoff criteria for fit indexes in covariance structure analysis: Conventional criteria versus new alternatives. Structural Equation Modeling: A Multidisciplinary Journal, 6(1), 1-55. doi: 10.1080/10705519909540118

Kline, R. B. (2011). Principles and practice of structural equation modeling (3. ed.). Nova Iorque: The Guilford Press.

Lamb, M. E. (1997). Father and child development: an introductory overview and guide. In M. E. Lamb (Org.), The Role of the Father in Child Development. New York, NY: John Wiley \& Sons, p.1-18.

Lima, J. A., \& Rodrigues, B. J. (2012). O envolvimento do pai num território educativo de intervenção prioritária. Revista AMAzônica, 5(8), 115-146. Recuperado de http://hdl.handle.net/10216/63882

Lundahl, B. W., Tollefson, D., Risser, H., \& Lovejoy, M. C. (2008). A meta-analysis of father involvement in parent training. Research on Social Work Practice, 18(2), 97-106. doi: 10.1177/1049731507309828

Marôco, J. (2010). Análise de equações estruturais. Pero Pinheiro: ReportNumber.

Marôco, J., \& Garcia-Marques, T. (2006). Qual a fiabilidade do alfa de Cronbach? Questões antigas e soluções modernas? Laboratório de Psicologia, 4(1), 65-90. doi: 10.14417/lp.763

Marsh, H. W., Hau, K. T., \& Wen, Z. (2004). In search of golden rules: Comment on hypothesis-testing approaches to setting cutoff values for fit indexes and dangers in overgeneralizing Hu and Bentler's (1999) findings. Structural Equation Modeling: A Multidisciplinary Journal, 11(3), 320-341. doi: 10.1207/s15328007sem1103_2

McAllister, F., Burgess, A., Kato, J. \& Barker, G., (2012). Fatherhood: Parenting programmes and policy - A critical review of best practice. London/ Washington D.C.: Fatherhood Institute/Promundo/MenCare.

Mehall, K. G., Spinrad, T. L., Eisenberg, N., \& Gaertner, B. M. (2009). Examining the relations of infant temperament and couples' marital satisfaction to mother and father involvement: A longitudinal study. Fathering, 7(1), 23-48. doi: 10.3149/fth.0701.23

Paquette, D., \& Dumont, C. (2013). The Father-Child Activation Relationship, Sex Differences, and Attachment Disorganization in Toddlerhood. Child Development Research, 2013, 1-9. doi: 10.1155/2013/102860

Paschoalick, M. M. (2008). Avaliando envolvimento paterno com filhos pré-escolares: Primeiros passos na construção de um instrumento. Proposta de pesquisa submetida ao Conselho Nacional de Desenvolvimento Científico e Tecnológico.

Paschoalick, M. M. (2009). Avaliando envolvimento paterno com filhos pré-escolares: passos intermediários na construção de um instrumento. Relatório final de pesquisa submetido ao Conselho Nacional de Desenvolvimento Científico e Tecnológico.

Pleck, J. H. (2007). Why could father involvement benefit children? Theoretical perspectives. Applied Development Science, 11(4), 196-202. doi: $10.1080 / 10888690701762068$

Pleck, J. H. (2010). Paternal involvement: Revised conceptualization and theoretical linkages with child outcomes. In Lamb, M. E. (Ed.), The Role of the Father in Child Development (2. ed.) (p. 58-93). Hoboken, NJ: John Wiley \& Sons. 
Pleck, J. H. (2012). Integrating father involvement in parenting research. Parenting: Science and Practice, 12, 243-253. doi: $10.1080 / 15295192.2012 .683365$

Stevenson, M. M., \& Crnic, K. A. (2013). Activative fathering predicts later children's behaviour dysregulation and sociability. Early Child Development Care, 183(6), 774-790. doi: 10.1080/03004430.2012.723441

Santis, L. de (2012). Envolvimento paterno: fatores associados com as diferenças nas percepções de mães e pais. Monografia de conclusão de curso em Psicologia, Universidade Federal de São Carlos, São Carlos, SP, Brasil.

Santis, L., \& Barham, E. J. (no prelo). Father involvement: Construction of a theoretical model based on a literature review. Temas em Psicologia.

Sarkadi, A., Kristiansson, R., Oberklaid, F. \& Bremberg, S. (2008). Fathers' involvement and children's developmental outcomes: A systematic review of longitudinal studies. ActaPediatrica, 97(2), 153-158. doi: 10.1111/j.1651-2227.2007.00572.x

Seward, R. R. \& Stanley-Stevens, L. (2014). Fathers, fathering, and fatherhood across cultures. Em Selin, H. (Ed.), Parenting Across Culture. (vol. 7) (p. 459-474). Springer.

Silva, N. C. B. (2011). Contexto familiar de crianças com síndrome de Down: interação e envolvimento paterno e materno. Tese de doutorado apresentado ao Programa de Pós-Graduação em Educação Especial da Universidade Federal de São Carlos, São Carlos, SP, Brasil.

Simões, R., Leal, I., \& Marôco, J. (2010). Paternal involvement in a group of fathers of elementary school children. Psicologia, Saúde E Doenças, 11(2), 339-356. Recuperado de http://www.scielo.mec.pt/pdf/psd/v11n2/v11n2a11.pdf

Schweizer, K. (2010). Some guidelines concerning the modeling of traits and abilities in test construction. European Journal of Psychological Assessment, 26(1), 1-2. doi: 10.1027/1015-5759/a000001

Tabachnick, B. G. \& Fidell, L. S. (2007). Using Multivariate Statistics (5. ed.). Boston, M.A.: Pearson Education, Inc.

Tautolo, E. S., Schluter, P. J., \& Paterson, J. (2015). Pacific father involvement and early child behavior outcomes: Findings from the Pacific Island families study. Journal of Child and Family Studies, 24, 3497-3505. doi: 10.1007/s10826-015-0151-5

Tornimbeni, S., Pérez, E., \& Olaz, F. (2008). Introducción a la psicometría (1. ed.). Buenos Aires: Paidós.

Volker, J. (2014). Paternal involvement: A review of the factors influencing father involvement and outcomes. TCNJ Journal of Student Scholarship, XVI, 1-8. Recuperado de http://joss.pages.tcnj.edu/files/2014/04/2014-Volker.pdf

\section{Sobre as autoras}

Ligia de Santis é Graduada e Mestre em Psicologia pela Universidade Federal de São Carlos (UFSCar). Atualmente é doutoranda em Psicologia na mesma instituição. Bolsista CAPES. Pesquisa o envolvimento paterno e as formas de mensuração do construto.

Elizabeth Joan Barham é Graduada em Psicologia pela Bishop's University, Mestre em Psicologia Social pela University of Waterloo e Doutora em Psicologia Social e de Desenvolvimento pela University of Guelph. Atualmente é docente no Programa de Pós-Graduação na Universidade Federal de São Carlos (UFSCar). Pesquisa questões de desenvolvimento adulto, com foco em habilidades metacognitivas e interpessoais de pessoas que cuidam de familiares dependentes (filhos ou parentes idosos).

Susana Coimbra é Licenciada em Psicologia pela Faculdade de Psicologia e de Ciências da Educação da Universidade de Coimbra, Mestre e Doutora pela Faculdade de Psicologia e de Ciências da Educação da Universidade do Porto, instituição em que é docente e investigadora. Pesquisa e orienta teses de mestrado e doutoramento no domínio da resiliência e solidariedade intergeracional em jovens e adultos.

Anne Marie Germaine Victorine Fontaine é Licenciada em Ciências Familiares e Sexologia pela Faculdade de Psicologia da Universidade Católica de Lovaina (Bélgica) e Doutora em Psicologia pela Faculdade de Psicologia e de Ciências da Educação da Universidade do Porto, na qual também é docente e investigadora. Pesquisa e orienta teses de mestrado e doutoramento com ênfase na área de Psicologia Diferencial (Differential Psychology). 